\title{
What are Ophthalmology Patients Asking Online? An Analysis of the Eye Triage Subreddit
}

This article was published in the following Dove Press journal:

Clinical Ophthalmology

\author{
Heba Mahjoub (1) \\ Arpan $\vee$ Prabhu $\mathbb{D}^{2}$ \\ Shameema Sikder (iD) 1,3 \\ 'School of Medicine, Johns Hopkins \\ University, Baltimore, MD, USA; \\ 2Department of Radiation Oncology, \\ UAMS Winthrop P. Rockefeller Cancer \\ Institute, Little Rock, AR, USA; ${ }^{3}$ The \\ Wilmer Eye Institute, Bethesda, MD, USA
}

Correspondence: Shameema Sikder The Wilmer Eye Institute, Bethesda, MD, USA

Email ssikderI@jhmi.edu
Importance: Ophthalmology patients are seeking medical advice on social media websites like Reddit, where users are able to post comments and discuss issues pertaining to different topics that are organized in 'subreddits'. Understanding which issues are most pertinent will guide ophthalmic providers in delivering more effective patient education.

Methods: This cross-sectional study assessed a systematic sample of the first 22 posts and their top 3 comments from each month since January 27th, 2019, the subreddit's creation. Information was gathered from reddit.com/r/eyetriage in October 2019 and analyzed in November 2019.

Main Outcomes: The posts were characterized by date and time, inclusion of an image, type, content, emotional tone, and number of upvotes and comments. The comments were categorized based on content, emotional tone, time of comment, and user background. Post and comment content codes were categorized in an iterative manner with differences resolved by author consensus. Categorical statistics were compiled.

Results: Two hundred posts and 456 comments were analyzed since the creation of $r$ / eyetriage, a forum created exclusively for patients to seek advice from health-care professionals. Twenty-six (13\%) of the total posts included an image. On average, comments received $1.76 \pm 2.17$ upvotes along with $4.50 \pm 4.47$ replies. The most common content codes among the posts were $42(21.0 \%)$ seeking diagnoses, 23 (11.5\%) surgical complications, and $13(6.50 \%)$ alternative medication options. Eighty-two (41\%) posts conveyed a clear emotional tone, most notably $11(13.4 \%)$ with anxiety and $10(12.2 \%)$ with worry. The top comments came from 165 (36.2\%) self-identified patients, 151 (33.1\%) optometrists, and $49(10.8 \%)$ ophthalmologists. The top comment codes for replies included $158(34.7 \%)$ with treatment advice, $70(15.4 \%)$ with advice deferred to follow-up appointment with other health-care specialists, and $60(13.2 \%)$ with sharing information.

Conclusions: Patients are asking ophthalmology-related questions on the Eye Triage subreddit, and they are more likely to receive information from other patients or optometrists than from self-identified ophthalmologists. When emotions were revealed, patients were often anxious and worried. Opportunities exist for ophthalmologists to take a more active role on this subreddit and help educate patients.

Keywords: Reddit, social media, patient education, ophthalmology, eye triage, subreddit

\section{Plain Language Summary}

Question: In 2019, what were ophthalmology patients seeking medical advice for on social media forums, such as r/eyetriage on Reddit?

Findings: This cross-sectional study of 200 posts and 456 comments found that most patients used r/eyetriage to seek diagnoses, acquire help with surgical complications, and discuss alternative medication options. Patients are more likely to receive information from other patients or optometrists than from ophthalmologists. 
Meaning: These results can guide ophthalmologists in improving patient education in the clinic and encourage their use of social media to regulate patient information online.

\section{Introduction}

Social media platforms such as Reddit, Twitter, and Facebook are increasingly being used by patients seeking health-care information. As online medical forums continue to develop, the number of patients interested in seeking help online also grows. ${ }^{1-3}$ Reddit is a rapidly growing website made for users to authentically and anonymously share stories, swap information, or engage in discussions with other users. The site consists of over 138,000 active communities, all divided into different topics, organized as "subreddits" with "r/topic" 10 The topics range from Harry Potter ( $r /$ harrypotter) and Korean pop culture ( $\mathrm{r} / \mathrm{kpop})$ to healthcare ( $\mathrm{r} /$ healthcare) and ophthalmology (r/Ophthalmology). Users can create a post on their own, and others can reply to, or comment on, that post. As of April 2018, Reddit had 330 million active users per month, which was equivalent to the number of users on Twitter at that time. ${ }^{4}$

Because of its popularity, Reddit has proven to be suitable for answering masses of health-related questions in a regulated manner. At the time of an outbreak of Nipah virus in India, Reddit was used to answer community members' questions with the help of several online moderators. ${ }^{5}$ With such a diverse user base, it represents a unique community with varying skillsets. Within Reddit, there are many medical-based communities where patients share their concerns, such as $\mathrm{r} /$ medical, which has 40,100 members. ${ }^{10}$ Multiple previous studies have been successful in analyzing how patients use Reddit to seek help with urinary incontinence, ${ }^{6}$ rheumatoid arthritis, ${ }^{7}$ HPV vaccination, ${ }^{8}$ and even underage electronic cigarette (JUUL) use. ${ }^{9}$ However, there is limited data regarding the activity of ophthalmology patients online. We wondered about patients' concerns and the type of information they were seeking most often. By understanding where the biggest gaps in online ophthalmology information exist, providers may be able to pre-emptively clarify confusions during an office visit. Furthermore, we can use this information to improve online sources of information in a systematically regulated manner. Ideally, patient education topics can be vetted by board-certified ophthalmologists or optometrists and archived onto Reddit for future patients to access. In the rapidly developing era of telemedicine, this online environment can become an ally to practicing clinicians.

\section{Methods}

In order to assess online information related to ophthalmology, the authors opted to analyze a patient-centered forum on Reddit. On January 27th, 2019, the subreddit $r$ / eyetriage (www.reddit.com/r/eyetriage/) was created exclusively for patients to seek advice from health-care professionals about their own health conditions. The community stresses that "online advice can never replace an actual medical examination", and all posts are moderated by a self-identified, anonymous ophthalmologist. ${ }^{10}$ This ophthalmologist either approves of comments to be posted or rejects them; however, the moderator does not actually respond to the posts, so patients are not always receiving feedback from an ophthalmologist. An example of a post that might be rejected is one that is not relevant to the eye. r/eyetriage has 896 actively subscribed members, some who ask questions, some who answer questions, and some who simply browse posts. All data in this study were collected from publicly available information online; thus, Institutional Review Board Approval was not needed. The methodology for this study was partially based on previous work done by Thomas et $\mathrm{al}^{11}$ where radiation therapy on Reddit was studied. When the authors discuss "posts", this refers to the original question or comment published for others to respond to. When the term "comment" is used, this signifies a reply to the original post. "Upvotes" indicate other users reacting positively to a post or comment; for example, a post with 3 upvotes insinuates less popularity among users than a post with 15 upvotes.

\section{Data Collection}

Reddit.com/r/eyetriage contains $1000+$ posts written since January 27th, 2019. We intended to analyze a random assortment of these posts in order to properly capture the breadth of users' thinking. Because there were hundreds of posts to sift through, we used random sampling to generate a representative normal population. We aimed to manually gather 200 posts, and r/eyetriage had 9 months of data. Hence, the first 22 posts from each month since the creation of r/eyetriage, along with their top 3 comments, were collected in late October 2019. If there were more than 3 comments, the comments with more upvotes were chosen over those with less upvotes, because the comments with more upvotes represented those more accessed and more 
favored by other users. Furthermore, if several users posted multiple comments, the top comment from each user was coded, rather than 3 from the same user, in order to avoid redundancy.

The original posts were manually sorted by time and date of post, post type, content code, emotional tone, and number of upvotes and comments. The comments were characterized based on content code, emotional tone, time of comment, and user background. As Reddit is an anonymous website, the user backgrounds were listed only when a user self-identified their occupational status. Each post and comment were coded based on subject matter according to the codes included in Table 1. Emotional codes were only included if the post contained clear evidence of the author's tone, such as explicitly stating an emotion (Table 2). One author (H.M.) initially manually reviewed and coded all posts, and a second author (A.V.P.) independently reviewed and coded the posts for inclusion in the final database. Infrequent code discrepancies were resolved through consensus (Table 1). This allowed consolidation of similar codes, for example, "glaucoma tube shunt complications" and "cataract complications" were converged to "surgical complications".

A similar method was used to code the comments, and this created a smaller list of 11 final codes (Table 3). When assessing for emotional tone of the comments, the authors felt that there were too few that were explicitly stated. Hence, they did not include emotional tone of comments. The users that replied often stated their role (Table 4), but when it was not stated in the comment itself, the authors skimmed that same user's replies in other posts in order to discover their role. If a user never clearly stated their position, this user was labeled "unspecified user".

\section{Results}

A total sample of 200 posts and 456 comments were analyzed since the origin of the $r$ /eyetriage subreddit on January 27th, 2019. Twenty-six (13\%) of the shared posts included an image. In total amongst the posts, there were 899 comments and 352 upvotes. However, in order to focus on the most relevant comments, only 456 comments were analyzed as they were among the top 3 comments. On average, comments received $1.76 \pm 2.17$ upvotes. Posts received a range of $0-30$ comments along with $0-27$ upvotes.

Upon author consensus, 32 unique post codes were created to encompass the content seen in the posts analyzed. The most common content codes among the posts
Table I Final Post Code Distribution

\begin{tabular}{|l|l|l|}
\hline Code & Frequency & Percent of Total \\
\hline Alternative medication options & 13 & 6.50 \\
Blind spot & 2 & 1.00 \\
Blurred vision & 9 & 4.50 \\
Cataracts & 1 & 0.50 \\
Clinic workflow & 1 & 0.50 \\
Color sensitivity & 1 & 0.50 \\
Comic & 1 & 0.50 \\
Contact lenses & 3 & 1.50 \\
Cornea & 2 & 1.00 \\
Dark spot & 7 & 3.50 \\
Double vision & 7 & 3.50 \\
Dry eye & 6 & 3.00 \\
Flashes & 6 & 3.00 \\
Floaters & 5 & 2.50 \\
Glasses & 7 & 3.50 \\
Glaucoma & 2 & 1.00 \\
Halos & 1 & 0.50 \\
Infection & 7 & 3.50 \\
Mechanism of medication & 1 & 0.50 \\
Medication side effects & 10 & 5.00 \\
Migraine & 1 & 0.50 \\
Need for triage & 1 & 0.50 \\
Ophthalmologic diagnostic tools & 5 & 2.50 \\
Photophobia & 3 & 1.50 \\
Prognosis & 6 & 3.00 \\
Retinal damage & 5 & 2.50 \\
Seeking diagnosis & 200 & 1.00 \\
Surgical complication & 21.0 \\
Surgical options & 11.5 \\
Trauma-induced vision deficit & 9 & 5.00 \\
Treatment cost & 4.50 \\
Vision loss & \\
Total & \\
\hline
\end{tabular}

were $42(21.0 \%)$ seeking diagnoses, $23(11.5 \%)$ surgical complications, and $13(6.50 \%)$ alternative medication options (Table 1). As an example of seeking diagnoses, one user shared a photo of their eye with the question, "Pinguecula?" (Supplementary Table 1). The medications most users asked about included FML-NEO eye drops, Lotemax, Lastacalf, pharmacologic vitreolysis, Occuvite, Lutein, Tobrex, and multiple glaucoma medications.

Eighty-two (41\%) posts conveyed a clear emotional tone, most notably $11(13.4 \%)$ with anxiety and 10 (12.2\%) with worry (Table 2). The top comments came from $165(36.2 \%)$ self-identified patients, 151 (33.1\%) optometrists, and 49 (10.8\%) ophthalmologists (Table 4). 
Table 2 Posts' Emotional Codes

\begin{tabular}{|c|c|c|}
\hline Emotional Tone & Frequency & Percent* \\
\hline Annoyed & 1 & 1.22 \\
\hline Anxious & 11 & 13.4 \\
\hline Concerned & 6 & 7.32 \\
\hline Confused & 2 & 2.44 \\
\hline Curious & 6 & 7.32 \\
\hline Depressed & 1 & 1.22 \\
\hline Desperate & 3 & 3.66 \\
\hline Distressed & 1 & 1.22 \\
\hline Dreadful & 1 & 1.22 \\
\hline Fearful & 6 & 7.32 \\
\hline Frustrated & 3 & 3.66 \\
\hline Helpless & 3 & 3.66 \\
\hline Humorous & 1 & 1.22 \\
\hline Impatient/restless & I & 1.22 \\
\hline Miserable & 1 & 1.22 \\
\hline Nervous & 1 & 1.22 \\
\hline Overwhelmed & 2 & 2.44 \\
\hline Paranoid & 3 & 3.66 \\
\hline Pessimistic & I & 1.22 \\
\hline Positive & 1 & 1.22 \\
\hline Preoccupied & I & 1.22 \\
\hline Scared & 5 & 6.10 \\
\hline Skeptical & 1 & 1.22 \\
\hline Stressed & 6 & 7.32 \\
\hline Suffering & I & 1.22 \\
\hline Uncomfortable & I & 1.22 \\
\hline Wonderous & 2 & 2.44 \\
\hline Worried & 10 & 12.2 \\
\hline Not available & 118 & - \\
\hline Total samples & 200 & 100 \\
\hline
\end{tabular}

Note: $*$ The percent was calculated relative to the 82 posts with available emotional codes.
The top comment codes included 158 (34.7\%) with treatment advice, 70 (15.4\%) with advice deferred to follow-up appointment with other health-care specialists, and 60 $(13.2 \%)$ with sharing information. After stratifying by 1st, 2nd, and 3rd top comments, we found that among the 1st top comment, $89(48.6 \%)$ contained treatment advice, $27(17.1 \%)$ contained thankfulness for response, and $43(34.7 \%)$ contained treatment advice, respectively (Table 3).

\section{Discussion}

We characterized 200 posts and 456 comments on the $\mathrm{r} /$ eyetriage subreddit and found that the majority of users were seeking a diagnosis in an anxious mood, and the comments came largely from patients or optometrists. The r/eyetriage subreddit is different from other communities in that it was created specifically for patients to communicate with ophthalmic providers, as opposed to $r$ / Ophthalmology. In contrast, on r/Ophthalmology, health professionals share interesting news or seek advice from other health professionals about their techniques and cases.

We saw that $42(21.0 \%)$ posts contained the most common post code, "seeking diagnosis" (Table 1). When patients seek diagnoses online, there is a risk of receiving inaccurate advice that might inappropriately increase their anxiety or even cause them to miss an important diagnosis. ${ }^{12}$ However, this risk does not deter patients from seeking diagnoses online. For example, from 2010 until $2019,58 \%$ of 16,979 posts about sexually transmitted diseases were seeking diagnoses on Reddit. ${ }^{13}$ Amongst

Table 3 Final Comment Code Distribution

\begin{tabular}{|c|c|c|c|c|}
\hline Code & $\begin{array}{l}\text { Frequency and \% } \\
\text { Total Ist Comment }\end{array}$ & $\begin{array}{l}\text { Frequency and \% } \\
\text { Total 2nd Comment }\end{array}$ & $\begin{array}{l}\text { Frequency and \% } \\
\text { Total 3rd Comment }\end{array}$ & $\begin{array}{l}\text { Frequency and \% } \\
\text { Total of all Replies }\end{array}$ \\
\hline $\begin{array}{l}\text { Advice deferred to follow-up } \\
\text { appointment with other health specialist }\end{array}$ & $38(20.8 \%)$ & $16(10.1 \%)$ & $16(13.9 \%)$ & $70(15.4 \%)$ \\
\hline Follow-up question about condition & 21 (11.5\%) & $18(11.4 \%)$ & $15(13.0 \%)$ & $54(11.8 \%)$ \\
\hline Follow-up question by original poster & $\mathrm{I}(0.55 \%)$ & 22 (13.92\%) & 7 (6.09\%) & $30(6.58 \%)$ \\
\hline Humor & I (0.55\%) & $0(0.00 \%)$ & $0(0.00 \%)$ & $\mathrm{I}(0.22 \%)$ \\
\hline Non-specific follow-up comment & $2(1.09 \%)$ & $7(4.43 \%)$ & $5(4.35 \%)$ & 14 (3.07\%) \\
\hline Sharing information & 29 (I5.9\%) & $19(12.03 \%)$ & $12(10.4 \%)$ & $60(13.2 \%)$ \\
\hline Symptom details & $\mathrm{I}(0.55 \%)$ & $23(14.6 \%)$ & II (9.57\%) & $35(7.68 \%)$ \\
\hline Thankfulness for response & $0(0.00 \%)$ & $27(17.1 \%)$ & $5(4.35 \%)$ & $32(7.02 \%)$ \\
\hline Treatment advice & 89 (48.6\%) & $26(16.5 \%)$ & $43(37.4 \%)$ & $158(34.7 \%)$ \\
\hline Comment deleted & $\mathrm{I}(0.55 \%)$ & $0(0.00 \%)$ & I $(0.87 \%)$ & $2(0.44 \%)$ \\
\hline Total & $183(100 \%)$ & $158(100 \%)$ & 115 (100\%) & $456(100 \%)$ \\
\hline
\end{tabular}


Table 4 User Roles and Distribution for Reply

\begin{tabular}{|c|c|c|c|c|}
\hline User Role & $\begin{array}{l}\text { Frequency and \% } \\
\text { Total Ist Replies }\end{array}$ & $\begin{array}{l}\text { Frequency and \% } \\
\text { Total 2nd Replies }\end{array}$ & $\begin{array}{l}\text { Frequency and \% } \\
\text { Total 3rd Replies }\end{array}$ & $\begin{array}{l}\text { Frequency and \% } \\
\text { Total of All Replies }\end{array}$ \\
\hline Optometrist & $89(48.6)$ & $28(17.7)$ & $34(29.6)$ & $151(33.1)$ \\
\hline Ophthalmologist & $28(15.3)$ & $9(5.70)$ & $12(10.4)$ & $49(10.8)$ \\
\hline Patient (non-original poster) & $21(11.5)$ & $26(16.5)$ & $23(20.0)$ & $70(15.4)$ \\
\hline Unspecified user & $17(9.29)$ & $10(6.33)$ & $12(10.4)$ & $39(8.55)$ \\
\hline Optometry student & $9(4.92)$ & $2(1.27)$ & $\mathrm{I}(0.87)$ & $12(2.63)$ \\
\hline Certified ophthalmologist assistant (COA) & $5(2.73)$ & $3(1.90)$ & $3(2.61)$ & II $(2.4 \mathrm{I})$ \\
\hline Technician & $3(1.64)$ & I $(0.63)$ & I $(0.87)$ & $5(1.10)$ \\
\hline Ophthalmic scribe & $2(1.09)$ & I $(0.63)$ & I $(0.87)$ & $4(0.88)$ \\
\hline Certified paraoptometric (CPO) & I $(0.55)$ & $0(0.00)$ & $0(0.00)$ & I $(0.22)$ \\
\hline Clinical research coordinator & I $(0.55)$ & $0(0.00)$ & $0(0.00)$ & I $(0.22)$ \\
\hline Engineer & I $(0.55)$ & $0(0.00)$ & $0(0.00)$ & I $(0.22)$ \\
\hline Internal medicine resident & I $(0.55)$ & I $(0.63)$ & $0(0.00)$ & $2(0.44)$ \\
\hline Occupational therapist & I $(0.55)$ & $0(0.00)$ & I $(0.87)$ & $2(0.44)$ \\
\hline Optician & I $(0.55)$ & I $(0.63)$ & $2(1.74)$ & $4(0.88)$ \\
\hline Orthoptist & I $(0.55)$ & I $(0.63)$ & $2(1.74)$ & $4(2.63)$ \\
\hline Patient (OP) & I $(0.55)$ & $73(46.2)$ & $21(18.3)$ & $95(20.8)$ \\
\hline Comment deleted & I (0.55) & $0(0.00)$ & I $(0.87)$ & $2(0.44)$ \\
\hline Ophthalmic registrar & $0(0.00)$ & $0(0.00)$ & I $(0.87)$ & I $(0.22)$ \\
\hline Optometry assistant & $0(0.00)$ & I $(0.63)$ & $0(0.00)$ & $\mathrm{I}(0.22)$ \\
\hline Orthoptic student & $0(0.00)$ & I (0.63) & $0(0.00)$ & $\mathrm{I}(0.22)$ \\
\hline Total & $183(100)$ & $158(100)$ & $115(100)$ & $456(100)$ \\
\hline
\end{tabular}

ophthalmic patients with nystagmus, one survey study showed that social media was the best source of information they received, even in comparison with in-person consultation with medical professionals. ${ }^{14}$

Interestingly, $13(6.50 \%)$ posts shared the third most prevalent post code, "alternative medication options" (Table 1). When reviewing 372 complaints by Canadian ophthalmology patients to the College of Physicians and Surgeons in Ontario, it was shown that roughly $75 \%$ were complaints related to clinical care and treatment. Among these, the largest subcategory was "communication" based complaints. ${ }^{15}$ Reddit allows users to post anonymously what they may be deterred from asking in clinic. Providers should elaborate on this information with patients at their appointments. Another potential solution is allowing online access to clinic notes, which has been shown to relieve some patients' confusion of their diagnosis or treatment plan after leaving an appointment. ${ }^{16}$ The majority of posts' emotional codes were negative feelings, including 11 anxious (13.4\%), 10 worried (12.2\%), 6 stressed $(7.32 \%), 6$ concerned $(7.32 \%), 6$ fearful $(7.32 \%)$, and 5 scared $(6.10 \%)$ posts (Table 2). This further indicates how addressing the Reddit community could relieve emotional burdens.
Ophthalmologists were the minority of self-identified users on the subreddit. When analyzing the users replying to patient posts, we saw that 165 were self-identified patients $(36.2 \%), 151$ were optometrists (33.1\%), and 49 were ophthalmologists (10.8\%) (Table 4). However, on Reddit, users are anonymous and roles are assigned only if the user self-identifies. This creates an air of uncertainty, as anyone on the internet could assume the role of a "board-certified ophthalmologist" and disseminate inaccurate information. One potential solution to this ambiguity is for interested ophthalmologists to self-identify themselves and guide patient users towards useful resources that they have previewed and trust. However, one must also consider the legal consequences of sharing medical advice online. The Ophthalmic Mutual Insurance Company (OMIC) warns that online medical guidance might be treated as "direct patient treatment"17 As such, it will be important for interested providers to describe the appropriate disclaimers needed when sharing their medical advice online.

When users replied to the original post, 158 (34.7\%) replies were providing treatment advice (Table 3). Supplementary Table 2 provides an example of a treatment advice-based comment, 
Impossible to say. Likely dryness; use artificial tears aggressively and see if that helps. If not, he could have developed a membrane behind the new lens that can be easily treated with a laser but this would cause more constant haze, not intermitting blurriness that worsens throughout the day.

This comment addresses many potential problems the patient might encounter and offers advice on treating each of these problems. When original posts were too vague, users were regularly inquisitive, as $54(11.8 \%)$ replies contained follow-up questions for the original patient (Table 3). One example is seen in Supplementary Table 2,

Do you change anything before this started happening? medications? work? stress? is it blurry up close, far away or both? does it change over the day? do you know if that little bit of prescription was long or short sighted?

Hence, providers were attempting to gather meaningful information in order to better understand the patients' needs. When the information provided remained insufficient, $70(15.4 \%)$ users redirected the original poster to follow-up with a provider in person (Table 3). For example, one provider wrote, "Honestly the information supplied is too vague for me to do anything with. Just keep your follow-up appointment and mention it to the doctor." This encouraged patients to follow-up with their physicians.

Besides using social media forums like Reddit for patient education, social media can also be used to help providers educate one another in clinical practice and academia. ${ }^{18}$ This can already be seen in a different subreddit, r/Ophthalmology, where the community was formed specifically for providers to seek advice from other providers. Online exchange of information was shown to change practice patterns among 58 of 178 users surveyed from a different online site, "Young Retina Forum". ${ }^{19}$ This can serve as an example of how Reddit can be used to create information-sharing communities for any subspecialty within ophthalmology.

The idea of using social media to guide patients and providers towards more accurate diagnoses or sharing treatment advice is quite exciting and novel. However, with all new advances, one must consider the potential negative consequences. Ultimately, social forums are meant to supplement a patient's primary source of healthcare. Though it is altruistic to desire maximal ease for our patients, we must also consider that regulations are in place to help promote patient safety. For example, in the past decade, there have been outbreaks of Acanthamoeba keratitis (AK) in both the USA and the UK..$^{20,21}$ In the UK, $90 \%$ of the affected patients were contact lens users, and many of the causes surrounded poor contact lens hygiene. Hence, it is important for ophthalmic providers to be able to teach proper contact lens hygiene prior to selling contacts. Furthermore, it is important for these patients to be able to regularly contact their providers in the case of complications. In the US, an outbreak in 2007 was analyzed by the CDC via surveys to ophthalmologists. It led to the FDA recalling a specific brand of contact lens solution. If social media was to take a stronger role in patients seeking diagnoses, many of these AK cases might have been missed and the contact lens solution would continue to be sold. Moving forward, we must keep in mind the potential downsides of a seemingly altruistic means of communication between patients and providers.

In considering the growth of social media as a provider tool, there will likely be push-back from providers who see it as encroaching on their field. It will be interesting to observe whether the new generation of providers will opt to encourage or reject an alternative pathway of healthcare that might disrupt workflow of patient care.

There is a rising need for medical professionals to advise patients online in a regulated manner. Incorporation of board-certified ophthalmic providers could ease patient burden while elucidating the source of most patients' concerns before leaving a clinical appointment. However, the authors respect the limited time of ophthalmologists; hence, it is more feasible to advocate for the creation of a curated section of frequently asked questions within the subreddit where patients can reference information that has previously been validated. It would also be helpful to direct patients towards other authentic sources, such as the "Ask an Ophthalmologist" section on EyeSmart.org. ${ }^{22}$ This realm has the potential to be advertised by various ophthalmologic societies, such as the American Academy of Ophthalmology, in an effort to improve patient education.

\section{Limitations}

There are several limitations to consider within our study. First, because of the nature of the website, all users share posts and comments anonymously. This means that a "self-identified ophthalmologist" cannot be confirmed, and hence the reporting of user roles might be inaccurate. This also means that several "unspecified users" might have been ophthalmologists or optometrists, but we 
would have no feasible means to gather this information. Second, post tones were only categorized if the users themselves stated an emotion, such as "I am frustrated". Thus, many posts sorted as "not available" might have had an emotional tone that we were unable to capture. In addition, there is no way to confirm whether a user's written emotion was not an exaggeration or embellishment to draw attention to their post. Furthermore, the population within the r/eyetriage subreddit might not be representative of the general population in an ophthalmic clinic. According to a 2016 survey completed by 4656 users, men make up $69 \%$ of Reddit users. ${ }^{23}$ Similarly, the difference in comments replied by optometrists versus ophthalmologists might just represent the fact that there are fewer ophthalmologists using Reddit. As such, those posting on r/eyetriage might differ from patients seeking help in ophthalmic clinics. In order for Reddit to be useful to the general public, it needs to be endorsed by certified providers and advertised to patients who might not otherwise know where to seek help online. Regardless, this study is important for guiding providers to begin curating a helpful, easy-to-use online patient education tool.

\section{Meeting Presentation Disclosure}

Data from this paper were presented in online oral presentation format at the 2020 Association for Research in Vision and Ophthalmology Meeting, which was meant to be held from May 3-7, 2020 in Baltimore, MD.

\section{Author Contributions}

All authors made a significant contribution to the work reported, whether that is in the conception, study design, execution, acquisition of data, analysis and interpretation, or in all these areas; took part in drafting, revising or critically reviewing the article; gave final approval of the version to be published; have agreed on the journal to which the article has been submitted; and agree to be accountable for all aspects of the work.

\section{Funding}

This research received no specific grant from any funding agency in the public, commercial or not-for-profit sectors.

\section{Disclosure}

The authors have no personal or institutional interest with regards to the authorship and/or publication of this manuscript. Arpan V Prabhu reports salary as resident physician from University of Arkansas for Medical Sciences, outside the submitted work. The authors report no other potential conflicts of interest for this work.

\section{References}

1. Cavazos-Rehg P, Grucza R, Krauss MJ, et al. Utilizing social media to explore overdose and $\mathrm{HIV} / \mathrm{HCV}$ risk behaviors among current opioid misusers. Drug Alcohol Depend. 2019;205:107690. doi:10. 1016/j.drugalcdep.2019.107690

2. Buyuk SK, Imamoglu T. Instagram as a social media tool about orthognathic surgery. Health Promot Perspect. 2019;9(4):319-322. doi:10.15171/hpp.2019.44

3. Waring ME, Libby BA, Moore Simas TA, et al. Delivering a post-partum weight loss intervention via facebook or in-person groups: protocol for a randomized feasibility pilot trial. JMIR Res Protoc. 2019;8(11):e15530. doi:10.2196/15530

4. Social Media Today. Reddit Now Has as Many Users as Twitter, and Far Higher Engagement Rates. Available from: https://www.social mediatoday.com/news/reddit-now-has-as-many-users-as-twitter-and -far-higher-engagement-rates $/ 521789 /$. Accessed December 23, 2019.

5. Cole J, Ezziane S, Watkins C. Rapid creation of an online discussion space ( $\mathrm{r} / \mathrm{nipah}$ ) during a serious disease outbreak: observational Study. JMIR Public Health Surveill. 2019;5(4):e13753. doi:10.2196/13753

6. Du C, Lee W, Moskowitz D, Lucioni A, Kobashi KC, Lee UJ. I leaked, then I Reddit: experiences and insight shared on urinary incontinence by Reddit users. Int Urogynecol J. 2019;31(2):243-248. doi:10.1007/s00192-019-04165-8

7. Chew C, Rebić N, Baldwin C, Amiri N, Proulx L, De Vera MA. "r/ Thritis", pregnancy, and parenting: a qualitative descriptive study of reddit forums to explore information needs and concerns of women with rheumatoid arthritis. ACR Open Rheumatol. 2019;1(8):485-492. doi:10.1002/acr2.11069

8. Amith M, Cohen T, Cunningham R, et al. Mining HPV vaccine knowledge structures of young adults from Reddit using distributional semantics and pathfinder networks. Cancer Control. 2020;27 (1):1073274819891442. doi:10.1177/1073274819891442

9. Zhan Y, Zhang Z, Okamoto JM, Zeng DD, Leischow SJ. Underage JUUL use patterns: content analysis of Reddit messages. $J$ Med Internet Res. 2019;21(9):e13038. doi:10.2196/13038

10. r/eyetriage. reddit. Available from: https://www.reddit.com/r/eyet riage/. Accessed November 22, 2019.

11. Thomas J, Prabhu AV, Heron DE, Beriwal S. Reddit and radiation therapy: a descriptive analysis of posts and comments over 7 years by patients and health care professionals. Adv Radiat Oncol. 2019;4 (2):345-353. doi:10.1016/j.adro.2019.01.007

12. Merchant RM, Asch DA. Protecting the value of medical science in the age of social media and "Fake News.". JAMA. 2018;320 (23):2415-2416. doi:10.1001/jama.2018.18416

13. Nobles AL, Leas EC, Althouse BM, et al. Requests for diagnoses of sexually transmitted diseases on a social media platform. JAMA. 2019;322(17):1712-1713. doi:10.1001/jama.2019.14390

14. Gummer SL, Evans M, Cygan A, et al. Evaluating the impact of information and support for people with nystagmus in the digital age: a patient and carer questionnaire study. Curr Eye Res. 2020;45 (6):713-717. doi:10.1080/02713683.2019.1696978

15. Saha R, Kabanovski A, Klejman S, Margolin E, Buys YM. Patients' complaints involving ophthalmologists in the province of Ontario, Canada: a 5-year review. Can J Ophthalmol. 2020;55(3):22-26. doi:10.1016/j.jcjo.2019.08.007

16. Wiley Online Library. Ophthalmology patients' interest in online access to clinic notes at three US clinics - Lee - 2017 -ophthalmic and physiological optics. Available from: https://onlinelibrary.wiley. com/doi/pdf/10.1111/opo.12367. Accessed November 22, 2019. 
17. American Academy of Ophthalmology. Advisory Opinion - Social Media and Professionalism.[February 24, 2018] Available from: https://www.aao.org/ethics-detail/advisory-opinion-social-mediaprofessionalism. Accessed January 10, 2020.

18. Tsui E, Rao RC. Navigating social media in \#ophthalmology. Ophthalmology. 2019;126(6):779-782. doi:10.1016/j.ophtha.2019.02. 015

19. Schechet SA, Pandya HK, Mehta MC. Social media collaboration of retinal physicians: A 'Young Retina Forum' survey. Eur J Ophthalmol. 2020;30(4):770-773. doi:10.1177/1120672119832186

20. Carnt N, Hoffman JJ, Verma S, et al. Acanthamoeba keratitis: confirmation of the UK outbreak and a prospective case-control study identifying contributing risk factors. $\mathrm{Br} J$ Ophthalmol. 2018;102 (12):1621-1628. doi:10.1136/bjophthalmol-2018-312544
21. Verani JR, Lorick SA, Yoder JS, et al. National outbreak of acanthamoeba keratitis associated with use of a contact lens solution, United States. J Emerg Infect Dis. 2009;15(8):1236-1242. doi:10.3201/ eid1508.090225

22. American Academy of Ophthalmology. Ask an Ophthalmologist. Available from: https://www.aao.org/eye-health/ask-ophthalmologist. Accessed October 3, 2020.

23. U.S. Reddit user share by gender Statista; 2016. Available from: https://www.statista.com/statistics/517155/reddit-user-distributionusa-gender/. Accessed December 26, 2019.

\section{Publish your work in this journal}

Clinical Ophthalmology is an international, peer-reviewed journal covering all subspecialties within ophthalmology. Key topics include: Optometry; Visual science; Pharmacology and drug therapy in eye diseases; Basic Sciences; Primary and Secondary eye care; Patient Safety and Quality of Care Improvements. This journal is indexed on PubMed

Submit your manuscript here: https://www.dovepress.com/clinical-ophthalmology-journal
Central and CAS, and is the official journal of The Society of Clinical Ophthalmology (SCO). The manuscript management system is completely online and includes a very quick and fair peer-review system, which is all easy to use. Visit http://www.dovepress.com/ testimonials.php to read real quotes from published authors. 